\title{
Breast cancer stem cells, cytokine networks, and the tumor microenvironment
}

\author{
Hasan Korkaya, Suling Liu, and Max S. Wicha
}

Comprehensive Cancer Center, Department of Internal Medicine, University of Michigan, Ann Arbor, Michigan, USA.

\begin{abstract}
Many tumors, including breast cancer, are maintained by a subpopulation of cells that display stem cell properties, mediate metastasis, and contribute to treatment resistance. These cancer stem cells (CSCs) are regulated by complex interactions with the components of the tumor microenvironment - including mesenchymal stem cells, adipocytes, tumor associated fibroblasts, endothelial cells, and immune cells - through networks of cytokines and growth factors. Since these components have a direct influence on CSC properties, they represent attractive targets for therapeutic development.
\end{abstract}

\section{Introduction}

Although much research has focused on the genetic abnormalities that initiate and drive cancer, there is now overwhelming evidence that the behavior of tumorigenic cells is also highly influenced by their microenvironment. In fact, decades of research have led to the view that tumors are in effect organ-like structures composed of numerous cell types whose interaction is required to drive and promote growth and metastasis. In some normal tissues, a small number of stem cells have the capacity to self renew as well as to differentiate into multiple lineages. There is increasing evidence that a similar hierarchy governs many tumors, including breast cancer. Cancer stem cells (CSCs) are operationally defined by their ability to initiate tumors in immunocompromised mice upon serial passage, a demonstration of self renewal, as well as their ability to differentiate into the non-self-renewing cells forming the tumor bulk (1-3). Analogous to the regulation of normal stem cells by their "niche," CSCs are regulated by, and in turn regulate, cells within the tumor microenvironment. These cells, including mesenchymal stem cells (MSCs), tissue-associated fibroblasts, and endothelial cells interact with CSCs via growth factor and cytokine networks (Figure 1). Furthermore, immunomodulatory cells, including $\mathrm{T}$ cells and macrophages, exert both inhibitory and stimulatory effects on CSCs and their progeny. The interaction between CSCs and their microenvironment provides new targets for therapeutic intervention. Since CSCs by virtue of their relative resistance to chemotherapy and radiation therapy may contribute to treatment resistance and relapse (4-6), the successful targeting of this cell population is critical. Strategies designed to specifically target the interaction between the CSC and its microenvironment represent an important approach to improving patient outcome.

Although tumor heterogeneity has been long recognized, the existence of cellular hierarchies of tumor cells resulting from differentiation has only been more recently appreciated. In 1997, Bonnet and Dick first reported the existence of a cellular hierarchy in human acute myelogenous leukemia (AML) that resembled the hierarchy found in normal hematopoiesis (7). At the apex of this hierarchy are leukemic stem cells identified by virtue of their expression of the stem cell markers, notably that they are $\mathrm{CD} 34^{+} /$

Conflict of interest: Max S. Wicha has financial holdings and is a scientific adviser for OncoMed Pharmaceuticals, is a scientific adviser for Pfizer, and has received research support from Dompe.

Citation for this article: J Clin Invest. 2011;121(10):3804-3809. doi:10.1172/JCI57099.
CD38-. These leukemic stem cells, but not the majority of leukemic blast cells, were able to transfer leukemia to immunosuppressed mice (7) More recent studies have suggested that the majority of $\mathrm{CD}_{3} 4^{+}$AMLs arise from progenitor cells rather than from hematopoietic stem cells, and that an individual leukemia may be driven by multiple clones of leukemia stem cells (8). These and other recent studies suggest that CSCs may be more heterogeneous than previously believed (9). Similar CSCs have subsequently been identified in many human solid malignancies including those of the breast, brain, colon, prostate, and pancreas (3,10-12). Subsequent studies have provided evidence that these CSCs mediate tumor metastasis and, by virtue of their relative resistance to radiation and chemotherapy, contribute to relapse following therapy $(5,6)$. This highlights the importance of understanding the pathways which regulate this cell population. As is the case with their normal counterparts, CSCs are regulated by intrinsic signals as well as extrinsic signals originating in the tumor microenvironment (13), and epigenetic as well as genetic changes which occur during carcinogenesis modulate these regulatory signals $(14,15)$. Stem cell regulatory pathways frequently dysregulated in tumor cells include the Notch, Hedgehog, Wnt, PI3K, NF-кB, and Jak/STAT pathways (16-20). These pathways are activated in some tumors via mutation of key regulatory elements, but in breast cancer, they are more often dysregulated by signals from the tumor microenvironment (21). Emerging evidence suggests that the tumor cell hierarchy, as well as multiple cellular elements in the microenvironment, co-evolve during the process of carcinogenesis (22). Bi-directional paracrine signals coordinately regulate tumorigenic cell populations including CSCs $(17,23-25)$. Tumorigenic cells in turn produce factors that attract and regulate the diverse variety of cell types that constitute the tumor microenvironment $(23,25)$. Interestingly, many of the pathways activated during tumor formation resemble those that mediate normal wound healing, including cytokine loops and the transcription factor NF- $\kappa \mathrm{B}(17)$. In addition, immunomodulatory cells provide both stimulatory and inhibitory influences on tumor development $(26,27)$. Here, we will review the links between inflammation and cancer, with an emphasis on cytokine networks and NF-кB signaling. We will also discuss how the diverse heterogeneity of cell types in the tumor microenvironment may impinge on these pathways to regulate the CSC population. Elucidation of these networks is of clinical importance since it may allow for the identification of novel therapeutic targets and the development of strategies to target breast cancer stem cell (BCSC) populations. 

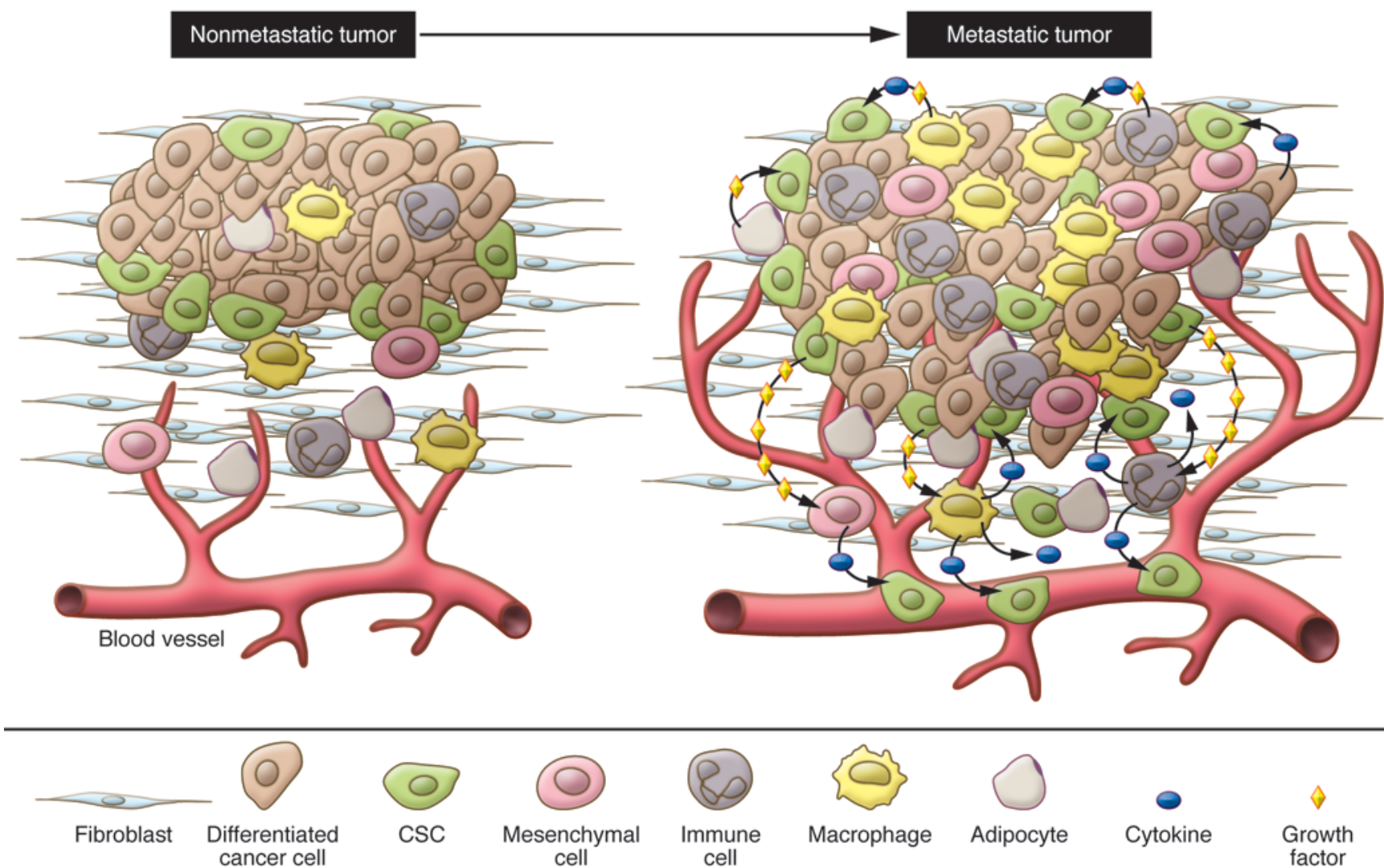

Figure 1

The tumor microenvironment. Elevated levels of cytokines and growth factors produced by tumor cells enhance the proliferation and survival of CSCs, induce angiogenesis, and recruit tumor-associated macrophages, neutrophils, and mast cells, which secrete additional growth factors, forming a positive feedback loop that promotes tumor cell invasion and metastasis.

\section{Cellular components of the tumor microenvironment}

Developing tumors recruit a diverse collection of cells that make up the microenvironment, and through iterative interactions both the tumor cell and its microenvironment co-evolve (22). Although early studies suggested that some of the cells in the tumor microenvironment contained mutations (28), more recent evidence suggests that mutations are limited to the tumorigenic cells, and that these modify the epigenetic program of other nontumorigenic cells in the tumor microenvironment $(21,28-30)$. The cells in the microenvironment in turn produce epigenetic changes in tumor cells reflected in their pattern of differentiation $(21,22)$. These reciprocal interactions are illustrated by changes in the tumor microenvironment that occur during evolution of pre-invasive ductal carcinoma in situ to invasive carcinoma of the breast, and involve sequential epigenetic changes in the tumor stromal microenvironment $(21,22)$.

In human breast cancers, mesenchymal cells may be recruited from the bone marrow (23) or from the normal breast stroma (25). As in tumor cells, ALDH1 expression identifies MSCs that are selectively recruited to sites of growing tumor, where they interact with BCSCs through cytokine loops involving IL-6 and CXCL7 (23). As illustrated in Figure 2, these cytokine loops stimulate the self renewal of BCSCs (23). Immunohistochemical analysis has confirmed the existence of such MSC/BCSC interactions in biopsies obtained from breast cancer patients (23). Expression of stem cell markers such as ALDH1 in breast cancer cells has been shown to be an independent predictor of poor outcome in women with breast cancer (2). In addition, MSCs have the ability to differentiate into adipocytes as well as tumor-associated fibroblasts, which also interact with and influence tumor cells (31).

The activation of fibroblasts and myofibroblasts was originally described in a study of wound healing by Gabbiani and Majno, who observed morphological changes in activated myofibroblasts as compared with quiescent tumor- and wound-associated fibroblasts (32). Based on the similarities between the wound healing process and cancer, both of which involve infiltration of inflammatory cells and activation of cytokine networks, it was proposed that malignant epithelial cells are "wounds that don't heal" (33). In an experimental mouse model, acute wounding in the mammary gland by dermal incision accelerated breast tumor growth and metastasis (34). Although the exact mechanisms remain unknown, paracrine signals from evolving tumors induce epigenetic changes in the surrounding stromal fibroblasts (35). Indeed, the gene expression profile of tumor-associated fibroblasts resembles that of wound-activated fibroblasts, and this profile is associated with poor prognosis $(36,37)$. Growth factors such as TGF- $\beta$ may be involved in these epigenetic changes, which lead to activation of fibroblasts (38). In addition, cytokines such as SDF-1 (also known as CXCL12) produced by breast carcinoma-associated fibroblasts (but not normal fibroblasts) may promote proliferation of tumor cells, which express the SDF-1 receptor CXCR4 (39). The level of expression of SDF-1 in serum has been associated with poor survival in breast cancer patients $(40,41)$. Other growth factors such as HGF, produced by mammary stromal cells, may also have a profound effect 


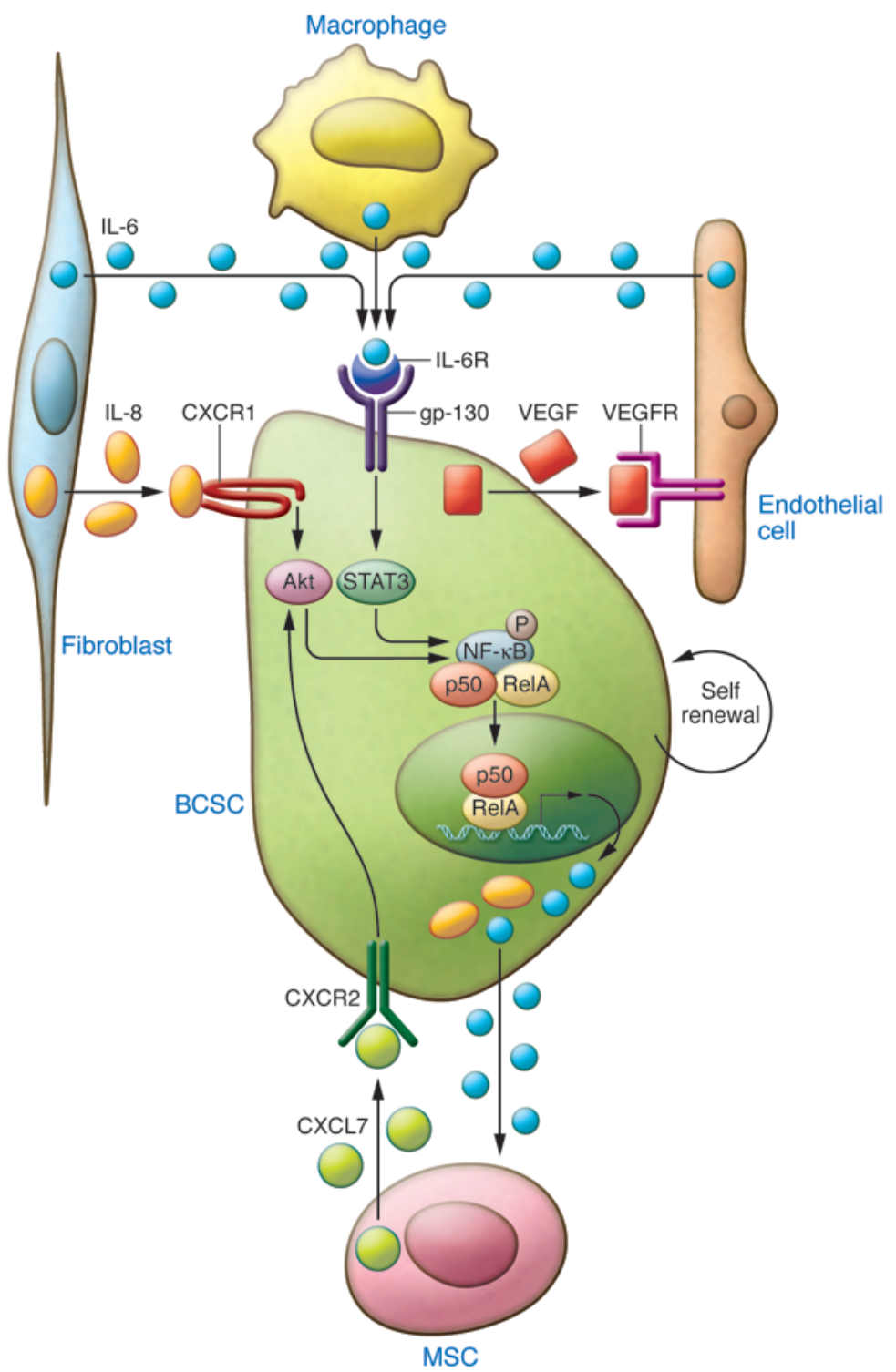

Figure 2

Cytokines secreted by cells in the tumor microenvironment regulate BCSC self renewal. Tumor-associated fibroblasts (TAFs) and macrophages (TAMs) and MSCs have been shown to secrete IL-6, IL-8, and CXCL7, which in turn activate STAT3/NF- $\mathrm{kB}$ signaling, leading to self renewal of BCSCs. This generates a positive feedback loop between the tumor microenvironment and tumor cells.

on developing mammary tumors (42). HGF provides a co-stimulatory signal to the Wnt pathway during colon carcinogenesis (43), although it remains to be determined whether similar pathways are involved in breast carcinogenesis. Other important growth factors produced by activated fibroblasts include the FGFs. It has recently been shown that estrogen regulates the BCSC population through a paracrine mechanism involving FGF9 (44). Additional factors produced by cells in the tumor microenvironment regulate tumor proliferation, invasion, and metastasis; these include IGF, PDGF, Wnt, Notch ligand, Hedgehog ligands, and MMPs (45-50).

The immune system exerts both inhibitory and stimulatory effects on breast tumors, and the balance of these effects may profoundly influence tumor growth. A description of tumor-mediated immunity is beyond the scope of this piece, and the reader is referred to recent excellent reviews on the subject (51-53). However, the importance of the immune system is illustrated by recent studies that have elucidated the mechanisms by which macrophages recognize and destroy tumor cells. Recent studies in human leukemia and lymphoma have suggested that tumor cells express the antigen CD47, which serves as a "don't eat me" signal to tumor-associated macrophages (54). At the same time, these cells express calreticulin, recognized by these macrophages as an "eat me" signal (55). Administration of a blocking antibody to CD47 induced macrophage phagocytosis of tumor cells in vitro and in mouse models $(55,56)$. Importantly, these researchers showed that leukemic stem cells as well as bulk tumor cells could be targeted by this approach. Although CD47 expression has been shown in breast cancer cells (57), it remains to be determined whether this antigen is also expressed on BCSCs and whether blocking this receptor would have utility in treating breast cancer.

Endothelial cells may play an important role in the tumor microenvironment by direct interaction with tumor cells as well as by their role in blood vessel formation. Endothelial cells constitute an important component of normal hematopoietic and neuronal stem cell niches $(58,59)$. In addition, cytokines produced by endothelial cells directly regulate CSCs $(60,61)$. It was proposed by Judah Folkman 40 years ago that angiogenesis, the process of new blood vessel formation, was required for tumor growth and metastasis (62). The role of tumor angiogenesis has been demonstrated in many preclinical models including breast cancer (63). This has led to the development of a number of antiangiogenic agents as cancer therapeutics. Angiogenesis is a complex process involving interaction between multiple cell types. Bone marrow-derived endothelial progenitor cells are attracted to tumors, where they differentiate into mature endothelial cells and form capillaries $(64,65)$. These newly formed blood vessels carry oxygen and nutrients to growing tumors, facilitating progression and metastasis (Figure 1). Interestingly, tumor vasculature is vastly different from normal vasculature, as illustrated by the finding that more than 1,000 genes are differentially expressed between them, including FGF receptors, MMPs, and JAK3 (60). Although preneoplastic lesions lack angiogenic capacity, transition from hyperplasia to neoplasia requires induction of angiogenesis, a process that may be regulated by NF- $\mathrm{kB}$ $(66,67)$. Tumors may also generate a vasculature via a process termed "vasculogenic mimicry," in which tumor cells transdifferentiate into vessel-forming endothelial-like cells. Two recent reports by Wang et al. and Ricci-Vitiani et al. demonstrated that glioblastoma CSCs are multipotent and can differentiate into endothelial cells, generat- 


\section{Table 1}

Breast tumor microenvironment constitutes diverse cell population which secretes various cytokines and growth factors

\section{Cell types}

Mesenchymal cells

Fibroblasts/myofibroblasts

Endothelial cells

Immune cells
Factors

CCL5, IL-6, CXCL5, IL-8

TGF- $\beta$, CXCL12, FGF, HGF,

IGF, PDGF, Wnt, MMPs

HGF, VEGF

IL-8, IL-6

\author{
Pathways activated \\ PI3K/AKT, NF-KB \\ $N F-\kappa B, P I 3 K / A K T$, \\ WNT/ $\beta$-catenin \\ PI3K/AKT, MAPK \\ PI3K/AKT, NF-KB, STAT3
}

\author{
References \\ 22-24, 79, 107 \\ $35,36,40,41,43,45-47,49$ \\ $60,65,75$ \\ $16,19,23,79,87$
}

ing their own vasculature $(68,69)$. Although many pro-angiogenic factors have been identified, VEGF is the primary mediator of this process (70), and as a result it has been the principal target of antiangiogenic therapies. A humanized monoclonal antibody targeting VEGF, bevacizumab, as well as two multi-kinase VEGF inhibitors, sorafenib and sunitinib, are currently approved for clinical use. These anti-angiogenic agents have shown utility in a number of tumor types including renal and colon cancers $(71,72)$. Bevacizumab was initially approved for use in metastatic breast cancer on the basis of reports demonstrating that it prolonged time to tumor progression (73). However, more recent studies (AVADO and RIBBON-1) have suggested that this effect is limited and that the addition of bevacizumab to cytotoxic chemotherapy failed to increase patient survival (74). These results are consistent with reports in mouse models that anti-angiogenic agents may accelerate local breast cancer invasion and distant metastasis $(75,76)$. Indeed, we have found that in mice bearing human breast cancer xenografts, these agents increase the CSC population through generation of tissue hypoxia (77). Antiangiogenic agents may also stimulate tumor growth by increasing HGF production by tumor stromal cells (78).

\section{Cytokine networks are important regulators of BCSCs}

The link between inflammation and cancer is an old concept that was first proposed by Virchow in 1864, when he observed that inflammatory cells frequently infiltrate tumor stroma (79). Considerable clinical evidence exists for links between inflammatory states and cancer development, including the association of ulcerative colitis, hepatitis $\mathrm{C}$, and chronic pancreatitis with cancers of the colon, liver, and pancreas, respectively (79). Levels of chronic inflammation as assessed by serum C-reactive protein or $\beta$-amyloid are correlated with risk of breast cancer recurrence in women after primary therapy (80). Chronic inflammation, as reflected in expression of these markers, may be mediated by cytokines including IL-1 $\beta$, IL-6, and IL-8 (53). Genetic polymorphisms in these cytokine genes predispose affected individuals to cancer (81). Cytokines generated by cells within the tumor microenvironment are shown in Table 1 . These inflammatory cytokines stimulate CSC self renewal, which then may promote tumor growth and metastasis $(24,82)$.

IL- 6 and IL- 8 have been implicated in both chronic inflammation and in tumor growth $(83,84)$. Within the tumor microenvironment, many cell types including mesenchymal cells, macrophages, and immune cells secrete both IL-6 and IL-8 (83). Furthermore, the serum levels of both of these cytokines have been associated with poor patient outcome in breast cancer $(85,86)$. In a variety of preclinical models, IL- 6 has been shown to promote tumorigenicity, angiogenesis, and metastasis (24, 87-89). The clinical relevance of these studies is supported by the demonstrated association between serum IL- 6 levels and poor patient outcome in breast cancer patients. IL- 6 has been shown to be a direct regulator of BCSC self renewal, a process mediated by the IL- 6 receptor/GP130 complex through activation of STAT3 (17). Utilizing mouse xenografts, we have recently demonstrated that bone marrow-derived MSCs are recruited to sites of growing breast cancers by gradients of IL-6 (23). IL-6 is a key component of a positive feedback loop involving these MSCs and BCSCs (23). Furthermore, Sethi et al. recently demonstrated that IL-6-mediated Jagged1/Notch signaling promotes breast cancer bone metastasis (90). These studies identify IL- 6 and its receptor as attractive therapeutic targets.

Utilizing gene expression profiling, we previously identified the IL-8 receptor CXCR1 as highly expressed on BCSCs and found that IL-8 was able to stimulate their self renewal (82). Blocking this receptor in mouse xenografts significantly reduced the number of BCSCs, leading to decreased tumorigenicity and metastasis. The production of inflammatory cytokines including IL- 6 and IL-8 is

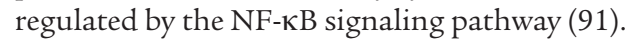

The NF- $\kappa \mathrm{B}$ pathway plays a crucial role in inflammation and carcinogenesis. The NF-kB family is composed of five related transcription factors: p50, p52, RelA (p65), c-Rel, and RelB $(92,93)$. In resting cells, NF- $\mathrm{KB}$ proteins are predominantly found in the cytoplasm,

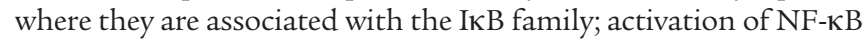
by diverse signals results in ubiquitin ligase-dependent degradation

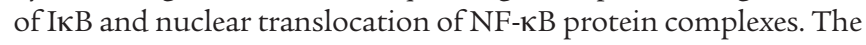
transcription of a number of cytokines, including IL- 6 and IL-8, is activated by NF-кB (91). In addition, a positive feedback loop has recently been shown to maintain a chronic inflammatory state in tumor cells. Interestingly, this loop involves the microRNA let7, as well as Lin28, a factor involved in embryonic stem cell self renewal (17). This feedback loop is maintained by IL- 6 through its activation of STAT3, which in turn activates NF- $\mathrm{KB}$ and its downstream targets Lin28 and let7. The specific role of IL-6 in maintaining this inflammatory loop in BCSCs has been recently demonstrated $(17,20)$. NF-кB may play an important role in normal breast physiology as well as carcinogenesis. In a HER2-neu model of mammary carcinogenesis, suppression of NF- $\mathrm{\kappa B}$ in mammary epithelium reduced the mammary stem cell compartment, resulting in a delayed onset of HER2-neu-induced tumors that displayed reduced angiogenesis and infiltration by macrophages (67). NF-кB has also been implicated in the regulation of mouse mammary stem cells during pregnancy. Elevated levels of progesterone during pregnancy induce the production of RANKL by differentiated breast epithelial cells. RANKL in turn stimulates breast stem cell self renewal via activation of NF- $\kappa \mathrm{B}$ in these cells $(94,95)$. The increased incidence of aggressive breast cancers associated with pregnancy (96) may result from activation of similar pathways in BCSCs $(94,95)$.

Epidemiologic studies demonstrate that obesity is associated with a significant increase in postmenopausal breast cancer $(97,98)$, 
which may be related to the known link between obesity and inflammation (99-102). Patients with type 2 diabetes mellitus have elevated levels of circulating proinflammatory cytokines including TNF- $\alpha$, IL-6, and C-reactive protein (103). In addition, these elevated levels of proinflammatory cytokines have been linked to NF- $\kappa$ B activation (104). The diabetes drug metformin reduces levels of circulating glucose, increases insulin sensitivity, and reduces insulin resistance-associated hyperinsulinemia. Interestingly, in preclinical mouse models, metformin has been shown to selectively inhibit BCSC self renewal, reducing the proliferation of these cells in breast tumor (105).

\section{Summary and clinical implications}

Many solid tumors, including breast cancer, are composed of heterogeneous cell populations that interact in complex networks. As is the case in developing organs, tumor cells interact with these diverse cellular elements, and these elements regulate the hierarchy of tumorigenic cells. Metastatic tumor cells also recreate complex cellular microenvironments at metastatic sites. Over 120 years ago, Paget proposed the "seed and soil" hypothesis of tumor metastasis (106). Reframed in a modern context, the seeds are the CSCs, and the soil is the rich microenvironment composed of diverse cell types which interact with tumor cells via growth factor and cytokine networks. These networks regulate CSCs and their progeny, which form the tumor bulk. Elucidation of these pathways may provide new targets for therapeutic development (Figure 2).
Researchers have already attempted to target some of these molecules and pathways, including the cytokines IL- 6 and IL- 8 and their receptors, IL-6R and CXCR1. Blockade of these pathways reduces the proliferation of BCSCs in mouse xenograft models of breast cancer (82). This results in reduction of tumor growth and metastasis in these models. NF- $\mathrm{KB}$ also represents an attractive target. Promising in vitro and preclinical studies using the NF- $\kappa \mathrm{B}$ inhibitor parthenolide (107) support the testing of this drug in early-stage clinical trials for the treatment of leukemia. These trials will determine whether the simultaneous targeting of CSCs and their microenvironment improves the efficiency of anticancer therapies.

\section{Acknowledgments}

We thank Pat LoRusso for discussions relevant to novel breast cancer therapeutic targets and their clinical implications and Craig T. Jordan for information pertaining to parthenolide trials. We also thank Shawn Clouthier for a critical review of this manuscript. This work was supported in part by NIH grants CA129765 and CA101860 as well as by funds from the American Association for Cancer Research (SU2C Dream Team Award), the Breast Cancer Research Foundation, and the Taubman Research Institute.

Address correspondence to: Max Wicha, Comprehensive Cancer Center, University of Michigan, 1500 E. Medical Center Drive, 7110 CC, Ann Arbor, Michigan 48109, USA. Phone: 734.936.1831; Fax: 734.647.9480; E-mail: mwicha@med.umich.edu.
1. Korkaya H, Paulson A, Iovino F, Wicha MS. HER2 regulates the mammary stem/progenitor cell population driving tumorigenesis and invasion. Oncogene. 2008;27(47):6120-6130.

2. Ginestier C, et al. ALDH1 is a marker of normal and malignant human mammary stem cells and a predictor of poor clinical outcome. Cell Stem Cell. 2007;1(5):555-567.

3. Al-Hajj M, Wicha MS, Benito-HernandezA, Morrison SJ, Clarke MF. Prospective identification of tumorigenic breast cancer cells. Proc Natl Acad Sci U S A. 2003;100(7):3983-3988.

4. Li X, et al. Intrinsic resistance of tumorigenic breast cancer cells to chemotherapy. J Natl Cancer Inst. 2008;100(9):672-679.

5. Liu H, et al. Cancer stem cells from human breast tumors are involved in spontaneous metastases in orthotopic mouse models. Proc Natl Acad Sci U S A. 2010;107(42):18115-18120.

6. Wicha MS, Liu S, Dontu G. Cancer stem cells: an old idea--a paradigm shift. Cancer Res. 2006; 66(4):1883-1890.

7. Bonnet D, Dick JE. Human acute myeloid leukemia is organized as a hierarchy that originates from a primitive hematopoietic cell. Nat Med. 1997; 3(7):730-737.

8. Qian Z, Fernald AA, Godley LA, Larson RA, Le Beau MM. Expression profiling of CD34+ hematopoietic stem/ progenitor cells reveals distinct subtypes of therapy-related acute myeloid leukemia. Proc Natl Acad Sci U S A. 2002;99(23):14925-14930.

9. Taussig DC, et al. Anti-CD38 antibody-mediated clearance of human repopulating cells masks the heterogeneity of leukemia-initiating cells. Blood. 2008;112(3):568-575

10. Li C, et al. Identification of pancreatic cancer stem cells. Cancer Res. 2007;67(3):1030-1037.

11. Collins AT, Berry PA, Hyde C, Stower MJ, Maitland NJ. Prospective identification of tumorigenic prostate cancer stem cells. Cancer Res. 2005; 65(23):10946-10951.

12. Singh SK, et al. Identification of a cancer stem cell in human brain tumors. Cancer Res. 2003;
63(18):5821-5828.

13. Vera-Ramirez L, et al. Gene-expression profiles, tumor microenvironment, and cancer stem cells in breast cancer: latest advances towards an integrated approach. Cancer Treat Rev. 2010;36(6):477-484.

14. Baylin SB, Herman JG. DNA hypermethylation in tumorigenesis: epigenetics joins genetics. Trends Genet. 2000;16(4):168-174.

15. Jaenisch R, Bird A. Epigenetic regulation of gene expression: how the genome integrates intrinsic and environmental signals. Nat Genet. 2003;33 suppl:245-254.

16. Korkaya H, et al. Regulation of mammary stem/progenitor cells by PTEN/Akt/beta-catenin signaling. PLoS Biol. 2009;7(6):e1000121.

17. Iliopoulos D, Hirsch HA, Struhl K. An epigenetic switch involving NF-kappaB, Lin28, Let-7 MicroRNA, and IL6 links inflammation to cell transformation. Cell. 2009;139(4):693-706

18. Liu S, et al. Hedgehog signaling and Bmi-1 regulate self-renewal of normal and malignant human mammary stem cells. Cancer Res. 2006;66(12):6063-6071.

19. Dontu G, Jackson KW, McNicholas E, Kawamura MJ, Abdallah WM, Wicha MS. Role of Notch signaling in cell-fate determination of human mammary stem/progenitor cells. Breast Cancer Res. 2004; 6(6):R605-R615.

20. Iliopoulos D, Jaeger SA, Hirsch HA, Bulyk ML, Struhl K. STAT3 activation of miR-21 and miR181b-1 via PTEN and CYLD are part of the epigenetic switch linking inflammation to cancer. $\mathrm{Mol}$ Cell. 2010;39(4):493-506.

21. Ma XJ, Dahiya S, Richardson E, Erlander M, Sgroi DC. Gene expression profiling of the tumor microenvironment during breast cancer progression. Breast Cancer Res. 2009;11(1):R7.

22. Polyak K, Haviv I, Campbell IG. Co-evolution of tumor cells and their microenvironment. Trends Genet. 2009;25(1):30-38.

23. Liu S, et al. Breast cancer stem cells are regulated by mesenchymal stem cells through cytokine networks. Cancer Res. 2011;71(2):614-624.

24 . Sansone P, et al. IL- 6 triggers malignant features in mammospheres from human ductal breast carcinoma and normal mammary gland. J Clin Invest. 2007;117(12):3988-4002.

25. Karnoub AE, et al. Mesenchymal stem cells within tumour stroma promote breast cancer metastasis. Nature. 2007;449(7162):557-563.

26. Mantovani A. Cancer: Inflaming metastasis. Nature. 2009;457(7225):36-37.

27. Yu H, Pardoll D, Jove R. STATs in cancer inflammation and immunity: a leading role for STAT3. Nat Rev Cancer. 2009;9(11):798-809.

28. Patocs A, et al. Breast-cancer stromal cells with TP53 mutations and nodal metastases. $N$ Engl J Med. 2007;357(25):2543-2551.

29. Trimboli AJ, et al. Pten in stromal fibroblasts suppresses mammary epithelial tumours. Nature. 2009;461(7267):1084-1091.

30. Campbell IG, Qiu W, Polyak K, Haviv I. Breastcancer stromal cells with TP53 mutations. N Engl JMed. 2008;358(15):1634-1635.

31. Pittenger MF, et al. Multilineage potential of adult human mesenchymal stem cells. Science. 1999; 284(5411):143-147.

32. Gabbiani G, Majno G. Dupuytren's contracture: fibroblast contraction? An ultrastructural study. Am J Pathol. 1972;66(1):131-146.

33. Dvorak HF. Tumors: wounds that do not heal. Similarities between tumor stroma generation and wound healing. NEngl J Med. 1986;315(26):1650-1659.

34. Stuelten $\mathrm{CH}$, et al. Acute wounds accelerate tumorigenesis by a $\mathrm{T}$ cell-dependent mechanism. Cancer Res. 2008;68(18):7278-7282.

35. Hu M, et al. Distinct epigenetic changes in the stromal cells of breast cancers. Nat Genet. 2005;37(8):899-905.

36. Farmer P, et al. A stroma-related gene signature predicts resistance to neoadjuvant chemotherapy in breast cancer. Nat Med. 2009;15(1):68-74.

37. Finak G, et al. Stromal gene expression predicts clinical outcome in breast cancer. Nat Med. 2008; 14(5):518-527.

38. Bierie B, Moses HL. Tumour microenvironment: TGFbeta: the molecular Jekyll and Hyde of cancer. 
Nat Rev Cancer. 2006;6(7):506-520.

39. Orimo A, et al. Stromal fibroblasts present in invasive human breast carcinomas promote tumor growth and angiogenesis through elevated SDF-1/ CXCL12 secretion. Cell. 2005;121(3):335-348.

40. Burger JA, Kipps TJ. CXCR4: a key receptor in the crosstalk between tumor cells and their microenvironment. Blood. 2006;107(5):1761-1767.

41. Chu QD, Panu L, Holm NT, Li BD, Johnson LW, Zhang S. High chemokine receptor CXCR4 level in triple negative breast cancer specimens predicts poor clinical outcome. I Surg Res. 2010;159(2):689-695.

42. Michieli $P$, et al. Targeting the tumor and its microenvironment by a dual-function decoy Met receptor. Cancer Cell. 2004;6(1):61-73.

43. Vermeulen L, et al. Wnt activity defines colon cancer stem cells and is regulated by the microenvironment. Nat Cell Biol. 2010;12(5):468-476.

44. Fillmore CM, et al. Estrogen expands breast cancer stem-like cells through paracrine FGF/Tbx3 signaling. Proc Natl Acad Sci US A. 2010;107(50):21737-21742.

45. Dufraine J, Funahashi Y, Kitajewski J. Notch signaling regulates tumor angiogenesis by diverse mechanisms. Oncogene. 2008;27(38):5132-5137.

46. Singer CF, et al. MMP-2 and MMP-9 expression in breast cancer-derived human fibroblasts is differentially regulated by stromal-epithelial interactions. Breast Cancer Res Treat. 2002;72(1):69-77.

47. Pietras K, et al. Inhibition of PDGF receptor signaling in tumor stroma enhances antitumor effect of chemotherapy. Cancer Res. 2002;62(19):5476-5484.

48. LeBedis C, Chen K, Fallavollita L, Boutros T, Brodt P. Peripheral lymph node stromal cells can promote growth and tumorigenicity of breast carcinoma cells through the release of IGF-I and EGF. Int J Cancer. 2002;100(1):2-8.

49. Hiscox S, Parr C, Nakamura T, Matsumoto K, Mansel RE, Jiang WG. Inhibition of HGF/SFinduced breast cancer cell motility and invasion by the HGF/SF variant, NK4. Breast Cancer Res Treat. 2000;59(3):245-254.

50. Dale TC, et al. Compartment switching of WNT-2 expression in human breast tumors. Cancer Res. 1996; 56(19):4320-4323.

51. Mantovani A, Romero P, Palucka AK, Marincola FM. Tumour immunity: effector response to tumour and role of the microenvironment. Lancet. 2008;371(9614):771-783

52. Mantovani A, Allavena P, Sica A, Balkwill F. Cancer-related inflammation. Nature. 2008; 454(7203):436-444.

53. Coussens LM, Werb Z. Inflammation and cancer. Nature. 2002;420(6917):860-867.

54. Chao MP, et al. Anti-CD47 antibody synergizes with rituximab to promote phagocytosis and eradicate non-Hodgkin lymphoma. Cell. 2010; 142(5):699-713.

55. Chao MP, et al. Calreticulin is the dominant prophagocytic signal on multiple human cancers and is counterbalanced by CD47. Sci Transl Med. 2010; 2(63):63ra94

56. Chao MP, et al. Therapeutic antibody targeting of CD47 eliminates human acute lymphoblastic leukemia. Cancer Res. 2011;71(4):1374-1384.

57. Manna PP, Frazier WA. CD47 mediates killing of breast tumor cells via Gi-dependent inhibition of protein kinase A. Cancer Res. 2004;64(3):1026-1036.

58. Calabrese $C$, et al. A perivascular niche for brain tumor stem cells. Cancer Cell. 2007;11(1):69-82

59. Sugiyama T, Kohara H, Noda M, Nagasawa T. Maintenance of the hematopoietic stem cell pool by CXCL12-CXCR4 chemokine signaling in bone marrow stromal cell niches. Immunity. 2006; 25(6):977-988

60. Bhati R, et al. Molecular characterization of human breast tumor vascular cells. Am J Pathol. 2008; 172(5):1381-1390.

61. Keith B, Simon MC. Hypoxia-inducible factors, stem cells, and cancer. Cell. 2007;129(3):465-472.

62. Folkman J. Tumor angiogenesis: therapeutic implications. NEngl J Med. 1971;285(21):1182-1186.

63. Huh JI, et al. Inhibition of VEGF receptors significantly impairs mammary cancer growth in C3(1)/Tag transgenic mice through antiangiogenic and non-antiangiogenic mechanisms. Oncogene. 2005;24(5):790-800.

64. Duda DG, et al. Evidence for incorporation of bone marrow-derived endothelial cells into perfused blood vessels in tumors. Blood. 2006;107(7):2774-2776.

65. Lyden D, et al. Impaired recruitment of bone-marrow-derived endothelial and hematopoietic precursor cells blocks tumor angiogenesis and growth. Nat Med. 2001;7(11):1194-1201.

66. Folkman J, Watson K, Ingber D, Hanahan D. Induction of angiogenesis during the transition from hyperplasia to neoplasia. Nature. 1989; 339(6219):58-61.

67. Liu M, et al. The canonical NF-\{kappa\}B pathway governs mammary tumorigenesis in transgenic mice and tumor stem cell expansion. Cancer Res. 2010; 70(24):10464-10473.

68. Wang R, et al. Glioblastoma stem-like cells give rise to tumour endothelium. Nature. 2010; 468(7325):829-833.

69. Ricci-Vitiani $L$, et al. Tumour vascularization via endothelial differentiation of glioblastoma stemlike cells. Nature. 2010;468(7325):824-828.

70. Holash J, Wiegand SJ, Yancopoulos GD. New model of tumor angiogenesis: dynamic balance between vessel regression and growth mediated by angiopoietins and VEGF. Oncogene. 1999;18(38):5356-5362.

71. Okines A, Cunningham D. Current perspective: bevacizumab in colorectal cancer--a time for reappraisal? Eur J Cancer. 2009;45(14):2452-2461.

72. Yang JC, et al. A randomized trial of bevacizumab, an anti-vascular endothelial growth factor antibody, for metastatic renal cancer. NEngl J Med. 2003; 349(5):427-434.

73. Miller K, et al. Paclitaxel plus bevacizumab versus paclitaxel alone for metastatic breast cancer. $N$ Engl JMed. 2007;357(26):2666-2676

74. Petrelli F, Barni S. Bevacizumab in advanced breast cancer: an opportunity as second-line therapy? [published online ahead of print December 14, 2010] Med Oncol. doi:10.1007/s12032-010-9773-z.

75. Paez-Ribes M, et al. Antiangiogenic therapy elicits malignant progression of tumors to increased local invasion and distant metastasis. Cancer Cell. 2009;15(3):220-231.

76. Ebos JM, Lee CR, Cruz-Munoz W, Bjarnason GA, Christensen JG, Kerbel RS. Accelerated metastasis after short-term treatment with a potent inhibitor of tumor angiogenesis. Cancer Cell. 2009; 15(3):232-239.

77. Santos SJ, Kakarala P, Heath AN, Clouthier SG, Wicha MS. Anti-angiogenic agents increase breast cancer stem cells via generation of tumor hypoxia. American Association for Cancer Research Abstract, 102nd Annual Meeting, Orlando, Florida, USA. 2011

78. Shojaei F, et al. HGF/c-Met acts as an alternative angiogenic pathway in sunitinib-resistant tumors. Cancer Res. 2010;70(24):10090-10100.

79. Balkwill F, Mantovani A. Inflammation and cancer: back to Virchow? Lancet. 2001;357(9255):539-545.

80. Pierce BL, et al. Elevated biomarkers of inflammation are associated with reduced survival among breast cancer patients. J Clin Oncol. 2009; 27(21):3437-3444.

81. Michaud DS, et al. Genetic polymorphisms of interleukin-1B (IL-1B), IL-6, IL-8, and IL-10 and risk of prostate cancer. Cancer Res. 2006;66(8):4525-4530.

82. Ginestier C, et al. CXCR1 blockade selectively targets human breast cancer stem cells in vitro and in xenografts. J Clin Invest. 2010;120(2):485-497.

83. Waugh DJ, Wilson C. The interleukin-8 pathway in cancer. Clin Cancer Res. 2008;14(21):6735-6741.
84. Scheller J, Rose-John S. Interleukin-6 and its receptor: from bench to bedside. Med Microbiol Immunol. 2006;195(4):173-183.

85 . Yao C, et al. Interleukin-8 modulates growth and invasiveness of estrogen receptor-negative breast cancer cells. Int J Cancer. 2007;121(9):1949-1957.

86 . Benoy $\mathrm{IH}$, et al. Increased serum interleukin-8 in patients with early and metastatic breast cancer correlates with early dissemination and survival. Clin Cancer Res. 2004;10(21):7157-7162.

87. Gao SP, et al. Mutations in the EGFR kinase domain mediate STAT3 activation via IL- 6 production in human lung adenocarcinomas. J Clin Invest. 2007;117(12):3846-3856.

88. Conze D, et al. Autocrine production of interleukin 6 causes multidrug resistance in breast cancer cells. Cancer Res. 2001;61(24):8851-8858.

89. Ara T, Declerck YA. Interleukin-6 in bone metastasis and cancer progression. Eur J Cancer. 2010; 46(7):1223-1231.

90. Sethi N, Dai X, Winter CG, Kang Y. Tumor-derived Jagged 1 promotes osteolytic bone metastasis of breast cancer by engaging notch signaling in bone cells. Cancer Cell. 2011;19(2):192-205.

91. Barnes PJ, Karin M. Nuclear factor-kappaB: a pivotal transcription factor in chronic inflammatory diseases. N Engl J Med. 1997;336(15):1066-1071.

92. Hoffmann A, Natoli G, Ghosh G. Transcriptional regulation via the NF-kappaB signaling module. Oncogene. 2006;25(51):6706-6716.

93. Moynagh PN. The NF-kappaB pathway. J Cell Sci. 2005;118(pt 20):4589-4592.

94. Joshi PA, et al. Progesterone induces adult mammary stem cell expansion. Nature. 2010; 465(7299):803-807.

95. Asselin-Labat ML, et al. Control of mammary stem cell function by steroid hormone signalling. Nature. 2010;465(7299):798-802.

96. Peck JD, Hulka BS, Poole C, Savitz DA, Baird D, Richardson BE. Steroid hormone levels during pregnancy and incidence of maternal breast cancer. Cancer Epidemiol Biomarkers Prev. 2002;11(4):361-368.

97. Guilherme A, Virbasius JV, Puri V, Czech MP. Adipocyte dysfunctions linking obesity to insulin resistance and type 2 diabetes. Nat Rev Mol Cell Biol. 2008;9(5):367-377.

98. Manabe Y, Toda S, Miyazaki K, Sugihara H. Mature adipocytes, but not preadipocytes, promote the growth of breast carcinoma cells in collagen gel matrix culture through cancer-stromal cell interactions. J Pathol. 2003;201(2):221-228.

99. Bennett NB, Ogston CM, McAndrew GM, Ogston D. Studies on the fibrinolytic enzyme system in obesity. J Clin Pathol. 1966;19(3):241-243.

100. Basen-Engquist K, Chang M. Obesity and cancer risk: recent review and evidence. Curr Oncol Rep. 2011; 13(1):71-76.

101.Jee SH, Kim HJ, Lee J. Obesity, insulin resistance and cancer risk. Yonsei Med J. 2005;46(4):449-455.

102. Bianchini F, Kaaks R, Vainio H. Overweight, obesity, and cancer risk. Lancet Oncol. 2002;3(9):565-574

103. Wellen KE, Hotamisligil GS. Inflammation, stress, and diabetes. J Clin Invest. 2005;115(5):1111-1119.

104.Cai D, et al. Local and systemic insulin resistance resulting from hepatic activation of IKK-beta and NF-kappaB. Nat Med. 2005;11(2):183-190.

105. Hirsch HA, Iliopoulos D, Tsichlis PN, Struhl K. Metformin selectively targets cancer stem cells, and acts together with chemotherapy to block tumor growth and prolong remission. Cancer Res. 2009; 69(19):7507-7511.

106. Fidler IJ. The pathogenesis of cancer metastasis: the 'seed and soil' hypothesis revisited. Nat Rev Cancer. 2003;3(6):453-458.

107. Hassane DC, et al. Chemical genomic screening reveals synergism between parthenolide and inhibitors of the PI-3 kinase and mTOR pathways. Blood. 2010;116(26):5983-5990. 